\title{
Long Memory Interdependency and Inefficiency in Bitcoin Markets
}

\author{
Eng-Tuck Cheah, Tapas Mishra, Mamata Parhi and Zhuang Zhang*
}

\begin{abstract}
We model cross-market Bitcoin prices as long-memory processes and study dynamic interdependence in a fractionally cointegrated VAR framework. We find (i) long-memory in both individual market and five-market systems depicting non-homogeneous informational inefficiency and (ii) a cointegration relationship with slow adjustment of shocks where uncertainty leaves a negative impact.
\end{abstract}

Key Words: Cross-market Bitcoin prices; Long-memory; Efficient market hypothesis; Fractionally cointegrated VAR.

JEL Classifications: C22, C32, E44, G14

${ }^{*}$ Cheah: Southampton Business School, University of Southampton, Highfield Campus, Southampton, SO17 1BJ, UK (E-mail: jeremy.cheah@soton.ac.uk). Mishra: Southampton Business School, University of Southampton, Highfield Campus, Southampton, SO17 1BJ, UK (E-mail: t.k.mishra@soton.ac.uk). Parhi: Roehampton Business School, University of Roehampton, UK. Email: Mamata.Parhi@roehampton.ac.uk. Zhang: Southampton Business School, University of Southampton, Highfield Campus, Southampton, SO17 1BJ, UK (E-mail: Zhaung.Zhang@soton.ac.uk). Acknowledgement: We are grateful to Prof. Pierre-Daniel Sarte, the editor and an anonymous referee for many helpful comments. We are solely responsible for any remaining errors. 


\section{Introduction}

Being both a valid form of payment and an alternative to governments-backed currency, Bitcoin has emerged, among the class of cryptocurrency, as the most popular digital money (Cheah and Fry, 2015; Pieters and Vivanco, 2017). Indeed, Bitcoin has been used to purchase legal and illegal good and services (Bohme et al., 2015) as more retailers are accepting Bitcoin for transactionary purposes. $^{1}$ While bulk of the existing literature is focused on the legal aspects and underlying blockchain technology, little is known about the adaptive capability of Bitcoin markets following changes in the regulations or arrival of a stochastic shock. ${ }^{2}$ This is important because a key element in the determination of Bitcoin prices is the assumption of full confidence of its users or what a user feels at a point of time on the expected market prices. This leads to an unrealistic frictionless supply and demand interactions in the Bitcoin market. Moreover, this 'feeling' is conditional on a variety of factors, such as, the general macroeconomic/financial conditions of the real economy. Therefore, Bitcoin markets are not completely 'memory-less' markets, rather are autoregressive in nature so that a shock in this market can leave a long-lasting impact on equilibrium prices. Quantifying this 'memory' and mapping its implications for market efficiency are very important in the context of the present research note.

Our premise appears to have strong support from the literature. Cheah and Fry (2015) and Katsiampa (2017) suggest that the recent volatility in Bitcoin prices is an outcome of market sentiments, where the latter can be associated with significant 'memory'. Therefore, fluctuations in Bitcoin markets are, at least, partially accounted for by the ability of the system to remember past shocks. However, a strong memory is not necessarily a boon for the Bitcoin market, because large volatility in Bitcoin prices and its strong persistence often undermine the stability and threaten the store value function of Bitcoin as a currency. ${ }^{3}$ Yet, the massive interests in Bitcoin investment appears to arise from the diversification strategy and hedging purposes in risk management. Indeed, Dyhrberg (2016a) found that Bitcoin is ideal for risk-averse investors in anticipation of negative shocks to the market, whereas Dyhrberg (2016b) found that Bitcoin could be used as a hedging asset against market specific risk.

The above discussions have a leading aim: to devise a robust mechanism that fully characterizes the profits in the Bitcoin market. Recalling that this market is virtually detached from the real economy, profits earned from the demand-supply equilibrium in the Bitcoin market is principally driven by price changes at a point of time. Theoretically, the way one models 'price changes' should capture various degrees of market-(in)efficiency. The nascent but growing literature on the subject employs $I(1) / I(0)$ framework, to test, whether the integration order $(d)$ for Bitcoin prices in a

\footnotetext{
${ }^{1}$ For example, the number of transactions per month using Bitcoin increased from 12,000 to 2.1 million from August 2010 to August 2014 and in December 2015, approximately 200,000 Bitcoin transactions were carried out per day (Polasik et al., 2015).

${ }^{2}$ Both the features are regularly present, for example, financial crisis, changes in political regimes and introduction of new regulations to control fluctuations in Bitcoin prices.

${ }^{3}$ See, for instance, a recent review in the Independent about the unprecedented fall in Bitcoin prices after a recent surge. http : //www.independent.co.uk/life - style/gadgets - and - tech/news/bitcoin - price - live - updates latest - value - exchange - rate - digital - cryptocurrency - futures - investment - a8147681.html.
} 
market $i$ at time $t$ (denoted as $Y_{i t}$ ), is equal to 1 or 0 (see equation 1 below).

$$
(1-L)^{d} Y_{i, t}=\epsilon_{t} ; \epsilon_{t} \sim \operatorname{iid}\left(0, \sigma^{2}\right)
$$

Rejection of a unit root null hypothesis (i.e., $d=1$ ) is taken as an indication of the presence of (weak-form) informational market efficiency in the series. In other words, the current price reflects all available information of its growth over time. In reality, it does not have to be so because the strict assumption of $I(1) / I(0)$ property can be contested on theoretical grounds. A significant amount of informational loss can occur unless we allow $d$ to assume a real value between 0 and 1. That is, we are left with very limited information on the adaptive capability of Bitcoin markets to stochastic shocks. This leads an investor to settle with an undesirable sub-optimal gains from risk-adjusted returns.

In the current research note, we relax this strict assumption and model Bitcoin prices in a longmemory framework. We examining their dynamic interdependence/co-movement patterns across markets, where for each market, $i$, we define

$$
(1-L)^{d} Y_{t}=\sum_{j=0}^{\infty}(-1)^{j}\left(\frac{d(d-1)(d-2) \ldots(d-j+1)}{j !}\right) Y_{t}
$$

In equation $2, \frac{d(d-1)(d-2) \ldots(d-j+1)}{j !}$ is the binomial coefficient defined for any real number $d$ and non-negative integer $j$. The impact of a shock to Bitcoin prices in this setting is not necessarily permanent or completely stationary. In line with Fama (1970) if Bitcoin prices display significant 'memory', it contradicts the efficient market hypothesis (EMH) in varying degrees.

To test whether cross-market Bitcoin markets display heterogeneous informational inefficiency, we model them as an interdependent system. In a single market context, Bouri et al. (2017) and Bariviera (2017) have provided some evidence of long-range dependence. However, rarely a market in a highly internationalized world is regarded as atomistic, in particular, the Bitcoin market whose cross-market mobility is relatively free from the strict limitations of monetary policy rules. A long-memory estimation with explicit modelling of cross-market interdependency can aid users with reliable information on Bitcoin price (co-)movements and efficiency at both individual market and system levels. An innovation in this paper thus, concerns identification of long-memory and characterization of cointegration relationship from a system perspective. Moreover, we hold that persistent uncertainty can exert a debilitating effect on the stability in Bitcoin markets. Accordingly, we examine the impact of uncertainty on price co-movements in this market. Recently developed fractionally cointegrated VAR (FCVAR) approach of Johansen and Nielsen (2010) forms the backbone of our empirical construct.

To investigate further, in Section 2, we present data characteristics and estimation method. Section 3 presents and discusses main results. Section 4 presents results from robustness exercise. Finally, Section 5 concludes with main findings of the paper along with relevant policy implications. 


\section{Data and methodology}

\subsection{Data characteristics}

We collect daily Bitcoin closing price data (between 27/11/2011-17/03/2017) for five developed markets (viz., Europe, USA, Australia, Canada and UK) from the aggregation website Bitcoin Charts (www.bitcoincharts.com). Data prior to 25/2/2014 are collected from Mt. Gox. Subsequent to Mt. Gox closure the remaining observations were collected from other exchange platforms such as Bitstamp (the largest European Bitcoin exchange), Zyado and LocalBitcoins.

Uncertainty plays a prominent role in shaping users' sentiment and the confidence in the Bitcoin prices. An ideal measure of uncertainty would be economic policy uncertainty of Baker et al. (2016) or financial uncertainty as in Ludvigson et al. (2016). However, daily data on these measures are not available. A close approximation, as suggested by Bloom (2009), is the estimated volatility of S\&P 500. ${ }^{4}$ We follow convention and estimate a class of $\operatorname{GARCH}(1,1)$ model. Assuming that shocks can leave an asymmetric impact on volatility, we estimate an asymmetric $\operatorname{GARCH}(1,1)$ specification of S\&P 500 and use the estimated volatility as a proxy for daily uncertainty for each Bitcoin market.

Figure 1 presents time series plots of Bitcoin closing prices. Notable trends emerge: the prices depict tendency of upward movement and visible evidence of co-movement with lead/lag (see for instance, the case of Australia and USA, and the GBP and Canadian closing prices). Descriptive statistics are presented in Table 1. We observe that Canadian closing prices display highest average prices (361.863) with a standard deviation of 423.213, whereas UK market (GBP closing prices) is among the lowest average (201.851) with a standard deviation of 228.289. Considering the percentile distribution of the closing prices, every market demonstrates large differences in mean; for instance, when we compare the 25th, 50th and 75th percentile distributions for Canada, the respective mean are $12.781,276.990,563.810$.

Table 1: : Descriptive Statistics of Bitcoin Closing Prices

\begin{tabular}{llllllllll}
\hline Variable & $\mathrm{N}$ & mean & $\mathrm{sd}$ & $\min$ & $\max$ & $\mathrm{cv}$ & $\mathrm{p} 25$ & $\mathrm{p} 50$ & $\mathrm{p} 75$ \\
\hline PRICES & & & & & & & & & \\
\hline EURO & 1057.000 & 236.821 & 253.342 & 1.521 & 1207.690 & 1.070 & 9.900 & 201.730 & 366.050 \\
USD & 1057.000 & 287.344 & 300.079 & 2.050 & 1285.330 & 1.044 & 12.890 & 226.150 & 448.340 \\
AUSTRALIA & 1057.000 & 360.467 & 389.687 & 2.089 & 2332.360 & 1.081 & 12.642 & 300.165 & 544.000 \\
GBP & 1057.000 & 201.851 & 228.289 & 1.355 & 1325.380 & 1.131 & 8.004 & 153.420 & 300.120 \\
CANADA & 1057.000 & 361.863 & 423.213 & 2.260 & 5405.410 & 1.170 & 12.781 & 276.990 & 563.810 \\
\hline \hline
\end{tabular}

To gain first hand knowledge of the presence of long-memory, we examine the autocorrelation function $(\mathrm{ACF})$ and the spectral density plots of the price data. A long-memory in prices can be expected if their ACFs decay asymptotically as a power law $\left(\tau^{-\alpha}\right.$ with $\left.\alpha<1\right)$ such that past prices leave a long-lasting impact on the present. This is precisely evident in Figure 2 (except GBP which appear to be stationary). The autocorrelation for the five prices is in the range of $0.125<\alpha<0.39$

\footnotetext{
${ }^{4}$ Bloom (2009) shows that stock market volatility closely mimics news-based uncertainty or macroeconomic/financial uncertainty at aggregate economy level.
} 
depicting remarkably strong memory. As an additional check, in Figure 3, we have presented the spectral density functions for each series. We expect that for a fractional process the spectral density functions would have mass concentrated near the origin. Indeed, this feature is prevalent across five markets, implying that the prices follow a long-memory process.

\subsection{Estimation}

\section{(A) Memory in an individual series}

Among various approaches to estimate the memory parameter $d$ in equation (2), we employ Phillips' (1999) modified log periodogram and Shimotsu and Phillips' (2005) exact local Whittle (ELW) estimators. The ELW makes no assumption about the presence of cointegration and is consistent both when cointegration is present and when it is absent. Moreover, this estimator is also applicable to both stationary and non-stationary case.

\section{(B) Memory in a system and its impact on equilibrium relationship}

To understand the dynamic nature of interdependence among Bitcoin markets, we employ a cointegrated vector autoregression (VAR) model which is able to capture fractional processes characterized by slowly decaying autocorrelation functions. In our case, the given vector of Bitcoin prices across markets, $Y_{i, t}$, is fractionally cointegrated if all the markets are integrated of the same order (say, $d$ ), and there is at least one linear combination of these components which fractionally integrated of order $b$, with $b<d$. Thus, the fractional cointegration assumes the existence of a common stochastic trend which is integrated of order $d$, and the short-term departures from the long-run equilibrium being integrated of order $d-b$. Johansen and Nielsen's (2010) fractionally cointegrated vector autoregressive (FCVAR) accommodates all these features and provide a robust estimation of the interdependent system. In our context, the FCVAR (see equations 3-8 below) is applied to the relative Bitcoin prices whereby local currencies are expressed with respect to GBP.

$$
\Delta^{b} Y_{t}=\alpha \beta^{\prime} L_{b} Y_{t}+\sum_{i=1}^{k} \Gamma_{i} \Delta^{b} L_{b}^{i} Y_{t}+\epsilon_{t},
$$

which we apply to $Y_{t}=\Delta^{d-b} X_{t}$ such that

$$
\Delta^{d} X_{t}=\alpha \beta^{\prime} L_{b} \Delta^{d-b} X_{t}+\sum_{i=1}^{k} \Gamma_{i} \Delta^{d} L_{b}^{i} X_{t}+\epsilon_{t} .
$$

As usual, $\epsilon_{t}$ is $p$-dimensional independent and identically distributed with mean zero and covariance matrix $\Omega$.

Moreover, $\alpha$ and $\beta$ are $p \times r$ matrices, where $0 \leq r \leq p$. The columns of $\beta$ represent the cointegrating relationships in the system, whereas the elements of $\beta^{\prime} X_{t}$ represent the long-run equilibria relationship among the variables in the system. Moreover, the coefficients in $\alpha$ represent the speed 
of adjustment towards equilibrium for each of the variables, whereas the parameters $\Gamma_{i}$ govern the short-run behaviour of the variables. We begin with the assumption that $d=b$ and that there is a constant term for the cointegration relationship in the model (see equation 4). This gives rise to:

$$
\Delta^{d} X_{t}=\alpha\left(\beta^{\prime} L_{d} X_{t}+\rho^{\prime}\right)+\sum_{i=1}^{k} \Gamma_{i} \Delta^{d} L_{d}^{i} X_{t}+\epsilon_{t}
$$

In the above, $\mu$ can correct for the fact that all initial values of $X_{t}$ are not observed. The model we estimate is

$$
\Delta^{d}\left(X_{t}-\mu\right)=L_{d} \alpha \beta^{\prime}\left(X_{t}-\mu\right)+\sum_{i=1}^{k} \Gamma_{i} \Delta^{d} L_{d}^{i}\left(X_{t}-\mu\right)+\epsilon_{t} .
$$

Note that $\beta^{\prime} \mu=-\rho^{\prime}$ represents the mean of the stationary cointegrating relations.

\subsection{Test of fractional cointegration}

We now test hypotheses to establish fractional cointegration. The two hypotheses are $H_{r}=$ $\operatorname{rank}(\Pi, \mu)=r$ and $H_{p}=\operatorname{rank}(\Pi, \mu)=p$. Let $L(d, b, r)$ be the profile likelihood function given rank $r$, where $(\alpha, \beta, \Gamma)$ have been concentrated out by regression and reduced rank regression (Johansen and Nielsen 2012). In this case the profile likelihood function is maximized both under the hypothesis $H_{r}$ and under $H_{p}$ and the LR test statistic is defined as follows:

$$
L R_{T}(q)=2 \log \left(\frac{L\left(\hat{d}_{p}, \hat{b}_{p}, p\right)}{L\left(\hat{d}_{r}, \hat{b}_{r}, r\right)}\right)
$$

Where $q=p-r, 0 l e r \leq p-1, L\left(\hat{d}_{p}, \hat{b}_{p}, p\right)=\max L(d, b, p)$ and $L\left(\hat{d}_{r}, \hat{b}_{r}, r\right)=\max L(d, b, r)$ Asymptotic distribution of $L R_{T}(q)$ depends on the parameter $b$. Hence if $0<b<0.5$ the cointegration relation is accounted as weak and therefore, to define the acceptance or rejection of critical value, chi-squared distribution with $q^{2}$ degree of freedom is used where $q=p-r$. For $0.50<b<2$ the cointegration relation is accounted as strong and the following LR test statistics is used:

$$
L R_{T}(q) \rightarrow \operatorname{Tr}\left\{\int_{0}^{1} d w(s) F^{\prime}(s)\left(\int_{0}^{1} F(s) F^{\prime}(s) d s\right) \int_{0}^{1} F(s) d w^{\prime}(s)\right\}
$$

The vector process $d W$ is the increment of ordinary vector Brownian motion of dimension $q=p-r$. The vector process $F$ depends on the deterministic similar to the cointegrated VAR model in Johansen (Johansen, 1995). Computer programs are available for computing asymptotic critical values and asymptotic $P$-values for the $L R$ rank tests in the fractional models. The results were obtained using the computer software by Nielsen and Morin (Nielsen \& Morin, 2014). 


\section{Results}

\section{1 (A) Long memory in individual Bitcoin market}

For each returns series we have computed both Phillips (1999) and the Two-step Exact Local Whittle (ELW) Estimator of Shimotsu and Phillips (2005) (see Table 2). The results are reported for three bandwidths, viz., $m=T^{0.6}, m=T^{0.7}$ and $m=T^{0.8}$ so that we can confirm that the presence of a long-memory is not sensitive to the choice of a specific bandwidth only. For the ELW estimator, the asymptotic standard error is computed as $(4 m)^{-1 / 2}$. For example, when $m=T^{0.6}$, the asymptotic standard error (S.E.) is 0.061 , whereas for $m=T^{0.7}$ and $m=T^{0.8}$, they are 0.043 and 0.030 , respectively. The results from the ELW estimation suggest that each series is significantly fractionally integrated. For instance, in case of $m=T^{0.6}$, the $\hat{d}$ varies between 0.416-0.546 across markets. Phillips' modified log periodogram estimates provide similar inferences although the estimates are slightly smaller than the EWL estimation. The standard errors for Phillip's estimates confirm that the estimated $d$ values are statistically significant at $5 \%$ level. Thus, estimation using both approaches conclude that individual price series are fractionally integrated.

Table 2: Estimates of fractional integration parameter, $d$ for returns $\left(100 * \ln \left(\frac{P_{t}}{P_{t-1}}\right)\right)$

\begin{tabular}{lcccccc}
\hline & $\hat{d}_{E L W}$ & $\hat{d}_{E L W}$ & $\hat{d}_{E L W}$ & $\hat{d}_{\text {Phillips }}$ & $\hat{d}_{\text {Phillips }}$ & $\hat{d}_{\text {Phillips }}$ \\
\hline & $m=T^{0.6}$ & $m=T^{0.7}$ & $m=T^{0.8}$ & $m=T^{0.6}$ & $m=T^{0.7}$ & $m=T^{0.8}$ \\
\hline GBP & 0.498 & 0.489 & 0.481 & 0.423 & 0.370 & 0.337 \\
USD & $(0.061)$ & $(0.043)$ & $(0.030)$ & $(0.024)$ & $(0.020)$ & $(0.019)$ \\
CANADA & 0.443 & 0.421 & 0.453 & 0.522 & 0.382 & 0.346 \\
& $(0.061)$ & $(0.043)$ & $(0.030)$ & $(0.020)$ & $(0.017)$ & $(0.014)$ \\
AUSTRALIA & 0.416 & 0.378 & 0.367 & 0.394 & 0.288 & 0.267 \\
EURO & $(0.061)$ & $(0.043)$ & $(0.030)$ & $(0.011)$ & $(0.009)$ & $(0.005)$ \\
& 0.546 & 0.560 & 0.586 & 0.682 & 0.590 & 0.521 \\
& $(0.061)$ & $(0.043)$ & $(0.030)$ & $(0.024)$ & $(0.014)$ & $(0.016)$ \\
& 0.535 & 0.508 & 0.522 & 0.474 & 0.358 & 0.321 \\
\hline
\end{tabular}

Note: $T=1057$. The standard errors are in brackets and are reported under each estimate. For $E L W$ the asymptotic standard error is $(4 m)^{-1 / 2}$, where the bandwidth $m$ is given as $m=T^{0.6}, m=T^{0.7}$ and $m=T^{0.8}$, respectively.

\section{2 (B) Evidence from system estimation: the FCVAR results}

We begin by determining lag, $k$, and rank of the 5-variables FCVAR system (four relative prices and uncertainty). Tables 3 and 4 summarize these results, respectively. A general-to-specific testing strategy of lag length in Table 3 revealed that the null hypothesis of zero lag was rejected until we reached $k=2$ following AIC criteria. The likelihood ratio (LR) test statistic for each $k$ with a $p$-value shows that for $k=2$, the $p$-value $=0.019$ and the $p$-value for the multivariate Ljung Box $Q$-test $(p m v Q)$ is 0.25 implying that for this lag length, there was no evidence of serial correlation. The estimated $d$ for $k=2$ is 0.510 implying that the Bitcoin markets possess a system-wide longmemory. We determine now the number of cointegrating rank of the FCVAR system. Results in 
Table 4 (meant to be read top to bottom) reveals that we reject the null of rank 0 against rank 5 . The sequential testing leads us to choose 4 where the $p$-value is 0.363 . For this rank, the estimated $d$ is 0.510 indicating that the Bitcoin market has a system long-memory and that such a memory implies high-inefficiency in the Bitcoin market.

In the final stage, we estimate an unrestricted FCVAR to test if the cross-market Bitcoin prices are fractionally cointegrated VAR (results are presented in Table 4). In general, the system depicts a cointegration pattern where the disequilibrium correction is slower. This can be tested by imposing a restriction on $d$. We expect a rejection of the null hypothesis if we believe that the system is actually characterized by fractional dynamics. The null hypothesis we test is $d=1$; i.e. that the model is a Cointegrated VAR. The alternative hypothesis is FCVAR. We impose an identification restriction which normalizes the $\beta$ matrix. This provides added benefit in terms of the interpretation of the equilibrium relations as it is more intuitive while analyzing the long-run dynamics of each Bitcoin variable separately.

Equation 9 summarizes the unrestricted FCVAR results corresponding to the pre-selected $k=$ 2 and $r=4$. The equilibrium relations are presented in equations 10-12. The estimate of $d$ is 0.510 with a standard error 0.018. The residuals appear well-behaved with no evidence of serial correlation; the Ljung-Box $Q$-test has a $p$-value of 0.251 (reported in parenthesis). On the righthand side of equation 9 , the estimated adjustment coefficients $\hat{\alpha}$ are shown in the vector preceding $\nu_{t}$, which is the stationary long-run equilibrium defined by $\nu_{t}=\hat{\beta}^{\prime}\left(X_{t}-\hat{\mu}\right)$. The coefficients that characterize this long-run equilibrium are normalized and presented in equations (10-12), with the

constant term given by $\hat{\beta}^{\prime} \mu$. This relation suggests that Euro/GBP relative price, for instance, are increasing in Canadian/GBP prices and decreasing in uncertainty.

Table 3: Lag-selection in the FCVAR Model

\begin{tabular}{lllllllll}
\hline$k$ & $r$ & $d$ & $b$ & $L R$ & $P$-value & $A I C$ & $B I C$ & $p m v Q$ \\
\hline 3 & 5 & 0.309 & 0.309 & 153.41 & 0.411 & -846.33 & -775.44 & 0.66 \\
2 & 5 & 0.510 & 0.510 & 316.72 & 0.019 & $-890.75^{*}$ & -844.22 & 0.54 \\
1 & 5 & 0.975 & 0.975 & 535.45 & 0.000 & -864.87 & $-865.44^{*}$ & 0.25 \\
0 & 5 & 1.022 & 1.022 & 0.00 & 0.000 & -822.39 & -856.90 & 0.00 \\
\hline \multicolumn{7}{c}{ Note: Total number of observations, $T=1057}$.
\end{tabular}

Table 4: Cointegration rank determination in the FCVAR model

\begin{tabular}{lllll}
\hline Rank & $d$ & $b$ & LR statistic & P-value \\
\hline 0 & 0.601 & 0.541 & 178.220 & 0.000 \\
1 & 0.524 & 0.564 & 100.015 & 0.000 \\
2 & 0.580 & 0.555 & 77.112 & 0.000 \\
3 & 0.596 & 0.574 & 40.319 & 0.000 \\
4 & 0.510 & 0.611 & 8.555 & 0.301 \\
5 & 0.589 & 0.605 & 1.018 & 0.555 \\
\multicolumn{5}{l}{ Note: Total number of observations, $T=1057}$.
\end{tabular}




$$
\begin{gathered}
\Delta^{\hat{d}}\left(\left[\begin{array}{c}
\text { Euro/GBP } \\
U S D / G B P \\
A U D / G B P \\
C A N A D A / G B P \\
\text { Uncertainty }
\end{array}\right]-\left[\begin{array}{c}
7.579 \\
6.305 \\
13.767 \\
-4.081 \\
30.317
\end{array}\right]\right)=L_{\hat{d}}\left[\begin{array}{c}
-0.100 \\
-0.099 \\
0.688 \\
-0.242 \\
0.371
\end{array}\right] \nu_{t}+\sum_{i=1}^{2}+\hat{\Gamma}_{i} \Delta^{\hat{d}} L_{\hat{d}}^{i}\left(X_{t}-\hat{\mu}\right)+\hat{\epsilon}_{t} \\
\hat{d}=0.510(p=0.018), Q_{\epsilon}(30)=281.050(p=0.251), \text { LogL }=886.390 \\
\text { Euro/GBP }=1.231+0.548 *(\text { Canadian } / G B P)-0.029 * \text { Uncertainty }+\nu_{t} \\
U S D / G B P=1.1771+0.804 *(\text { Canadian } / G B P)-0.038 * \text { Uncertainty }+\nu_{t} \\
A U D / G B P=2.649+0.762 *(\text { Canadian } / G B P)-0.152 * \text { Uncertainty }+\nu_{t}
\end{gathered}
$$

\section{Robustness}

\subsection{Identification of break-points}

How robust are our results to the consideration of a break point in the sample? It is well-known that structural breaks can leave a long and non-stationary impact on a time series, leading to a wrong inference on the overall stability of the parameters. As Bitcoin prices display significant fluctuations, these may point to both shifting mean and variances over time (see Figure 1). Therefore, it is necessary to identify the significant change points in the data. A multiple-break test with unknown change point is suitable for our purpose, because imposition of an exogenous known break-point may further induce measurement bias. We use Andrews' (1993) critical value and perform a Sup-Wald test in each Bitcoin market.

The sup-Wald test identified 2/12/2013 as the break point for Euro, USD and Australia, whereas for Canada and the UK, the identified break point is 25/02/2014; the Sup-Wald values are 42.850 and 47.210, respectively with a p-value of 0.000 implying a rejection of no-break point in the data. Given that Bitcoin markets are highly integrated and are largely detached from the dynamics of the real economy, many markets may share common dates of break-points as is the case in our context. Do these identified change points correspond to any known historical antecedents? ${ }^{5}$ Our investigation revealed that in the first week of December, 2013, the People's Bank of China

\footnotetext{
${ }^{5}$ Many thanks to the anonymous referee for pointing this out.
} 
prohibited Chinese financial institutions from using Bitcoins. After the announcement, the value of Bitcoins dropped, and Baidu no longer accepted Bitcoins for certain services. ${ }^{6}$ Similarly, in the early February, 2014, one of the largest Bitcoin exchanges, Mt. Gox, suspended withdrawals citing technical issues. By the end of the month, Mt. Gox had filed for bankruptcy protection in Japan amid reports that 744,000 Bitcoins had been stolen. ${ }^{7}$

\subsection{FCVAR implementation after break-point identification}

Having identified the two break-points for different markets, we now present estimates of univariate long-memory. Prior to 2/12/2013, the ELW estimates of $d$ for bandwidth 0.6 are 0.535 for Euro, 0.458 for USD and 0.513 for Australia. For UK and Canada (before 25/02/2014), the estimates are 0.499 for GBP and 0.586 for Canadian dollar. In the post-break point period, we found that $\hat{d}$ are 0.285 for Euro, 0.612 for Australia, 0.354 for USD, 0.292 for GBP and 0.148 for Canada. While both sub-sample periods depict significant long-memory, the post break point sample produces lower persistence.

Our next step is to estimate an unrestricted FCVAR for the two sub-sample periods. For ease of reference and to minimizing repetition, we denote the samples before (after) 2/12/2013 and 25/02/2014 as pre- (post)-break point period. As before, we need to define the dimension of the FCVAR system. For both periods, the AIC criterion led to us to choose $k=1$ as the dimension of the system. By setting $k=1$, we now determine the cointegration rank of the system. The likelihood ratio test for sequential rank testing (i.e., testing the null hypothesis of no cointegration relationship against the highest order cointegration relationship) led us to choose $r a n k=4$ for both periods. However, the system long-memory estimates produced heterogeneous values for $d$; the $\hat{d}$ is 0.524 in the pre-change point sample. For this period, we now estimate an unrestricted FCVAR having set $k=1$ and $r a n k=4$. The $\hat{d}=0.323$ with a $p$-value of 0.031 ). If the FCVAR system presents no evidence of serial correlation, then the above estimates of cointegration rank and the $d$ would be unbiased. We find that the in our case, the multivariate $Q$-statistic $=622.391$ with a $p$-value of 0.122 implying that the null hypothesis of no serial correlation in the data could not be rejected. In other words, the estimates from the unrestricted FCVAR for the pre-break point sample are reliable.

We carry out similar exercise for the post-break point sample. With $k=1$ and $\operatorname{rank}=4$ (determined earlier), the estimation of unrestricted FCVAR produced the following results. The $\hat{d}$ $=0.446(p=0.004)$ and the $p$-value of the multivariate $Q$-statistic $=0.28$. Once again, the unrestricted FCVAR estimation confirms that there is a system long-memory and that the cointegration relationship accommodates a disequilibrium correction component that is slowly-adjusting to the long-run mean. Comparing the results from both pre- and post-break points lead us to infer that the Bitcoin markets possess significant long-memory and are fractionally cointegrated. Although, the

\footnotetext{
${ }^{6}$ See https : //www.theguardian.com/technology/2013/dec/18/bitcoin - plummets - china - payment processors - digital - cryptocurrencyfordetails.

${ }^{7}$ See https : //www.theguardian.com/technology/2014/feb/28/bitcoin-mtgox-bankruptcy-japan for details.
} 
presence of structural break impacted the magnitude of $\hat{d}$, yet, we found no evidence of insignificant system long-memory.

\section{Conclusions and implications}

In this note, we have proposed a new mechanism to understand dynamic interdependence of Bitcoin prices in a cross-market context. Due to the unique nature of the Bitcoin market (especially, the feature that it is least affected by the regulations of the real economy), it is highly probable that the determination of equilibrium prices in this market can be significantly guided by the strength of the 'memory'. This long-memory, for which we found significant evidence across markets, can cause a 'system failure' in the event of the introduction of new regulations in the financial markets. It appears to be the case because the spiraling new highs of Bitcoin prices has forced many governments (for example, India) to impose certain regulatory restrictions on the acceptance of Bitcoin as a legal currency (see the following link for details: https://www.coindesk.com/bitcoin-not-legaltender-in-india-finance-minister-says/).

Our results also indicate that Bitcoin markets are moderate to highly inefficient where the estimated memory in prices can help investors capture speculative profits. There is also an evidence of slow adjustment of disequilibrium error implying that the interdependent Bitcoin markets can be significantly affected by movement of a stochastic shock. Finally, we find that uncertainty has a general negative effect on Bitcoin markets. The overall policy implication is the following. While there is a general claim of virtual detachment of Bitcoin market from the real economy leading to the assumption that economic fluctuations should have least impact on its equilibrium price determination, we showed that it is actually inherently tied to the dynamics of the real economic system. The observed inefficiency in the Bitcoin market (as reflected by our system memory estimates) can therefore be, at least partially, regulated and tightly interlinked to the real economy.

\section{References}

[1] Andrews, D.,1993.Tests for Parameter Instability and Structural Change with Unknown Change Point., Econometrica 61(4): 821856.

[2] Baker, S., Bloom, N. and Davis, S.J., 2016. Measuring economic policy uncertainty. Quarterly Journal of Economics 131(4), 1593-1636.

[3] Bloom, N., 2009. The impact of uncertainty shocks. Econometrica 77 (3), 623-685.

[4] Bohme, R., N. Christin, Edelman, B. and Moore, T., 2015. Bitcoin: Economics, technology and governance. Journal of Economic Perspectives 29(2), 213-238.

[5] Bariviera, A.F., 2017. The Inefficiency of Bitcoin Revisited: The Dynamic Approach. Economics Letters 161, 1-4. 
[6] Bouri, E., Azzi, G., Dyhrberg, A.H., 2017. On the return-volatility relationship in the Bitcoin market around the price crash of 2013. Economics 11(2), 116.

[7] Cheah, E.T. and Fry, J.M., 2015. Speculative bubbles in Bitcoin markets? An empirical investigation into the fundamental value of Bitcoin. Economics Letters 30, 32-36.

[8] Dyhrberg, A.H., 2016a. Bitcoin, gold and the dollar - A GARCH volatility analysis. Finance Research Letters 16, 85-92.

[9] Dyhrberg, A.H.,2016b. Hedging capabilities of Bitcoin. Is it the virtual gold? Finance Research Letters 16, 139-144.

[10] Fama, E.F., 1970. Efficient capital markets: A review of theory and empirical work. Journal of Finance 25(2), 383-417.

[11] Johansen, S., 1995. Likelihood-Based Inference in Cointegrated Vector Autoregressive Models. New York:

[12] Johansen, S. and Nielsen, M.O., 2010. Likelihood inference for a fractionally cointegrated vector autoregressive model, Econometrica 80, 2667-2732.

[13] Katsiampa, P., 2017. Volatility estimation for Bitcoin: A comparison of GARCH models. Economics Letters 158, 3-6.

[14] Ludvigson, S.C., Mai, S. and S. Ng, 2016. Uncertainty and Business Cycles: Exogenous Impulse or Endogenous Response?, http://www.sydneyludvigson.com/working-papers/.

[15] Nielsen, M. and Morin, L., 2014. FCVARmodel.m: a Matlab software package for estimation and testing in the fractionally cointegrated VAR model, QED working paper 1273, Queen's University.

[16] Phillips, Peter C.B., 1999. Unit Root Log Periodogram Regression. Unpublished working paper No. 1244, Cowles Foundation for Research in Economics, Yale University. http://cowles.econ.yale.edu/P/cd/d12a/d1244.pdf

[17] Pieters, G. and Vivanco, S., 2017. Financial regulations and price inconsistencies across Bitcoin markets. Information Economics and Policy 39, 1-14.

[18] Polasik, M., Piotrowska, A.I., Wisniewski, T.P., Kotkowski, R. and Lightfoot, G., 2015. Price Fluctuations and the Use of Bitcoin: An Empirical Inquiry. International Journal of Electronic Commerce 20(1), 9-49.

[19] Shimotsu, K. and Phillips, P., 2005. Exact Local Whittle Estimation of Fractional Integration. Annals of Statistics 33(4), 1890-1933. 


\section{Appendix}

Figure 1: Trend in Closing Prices
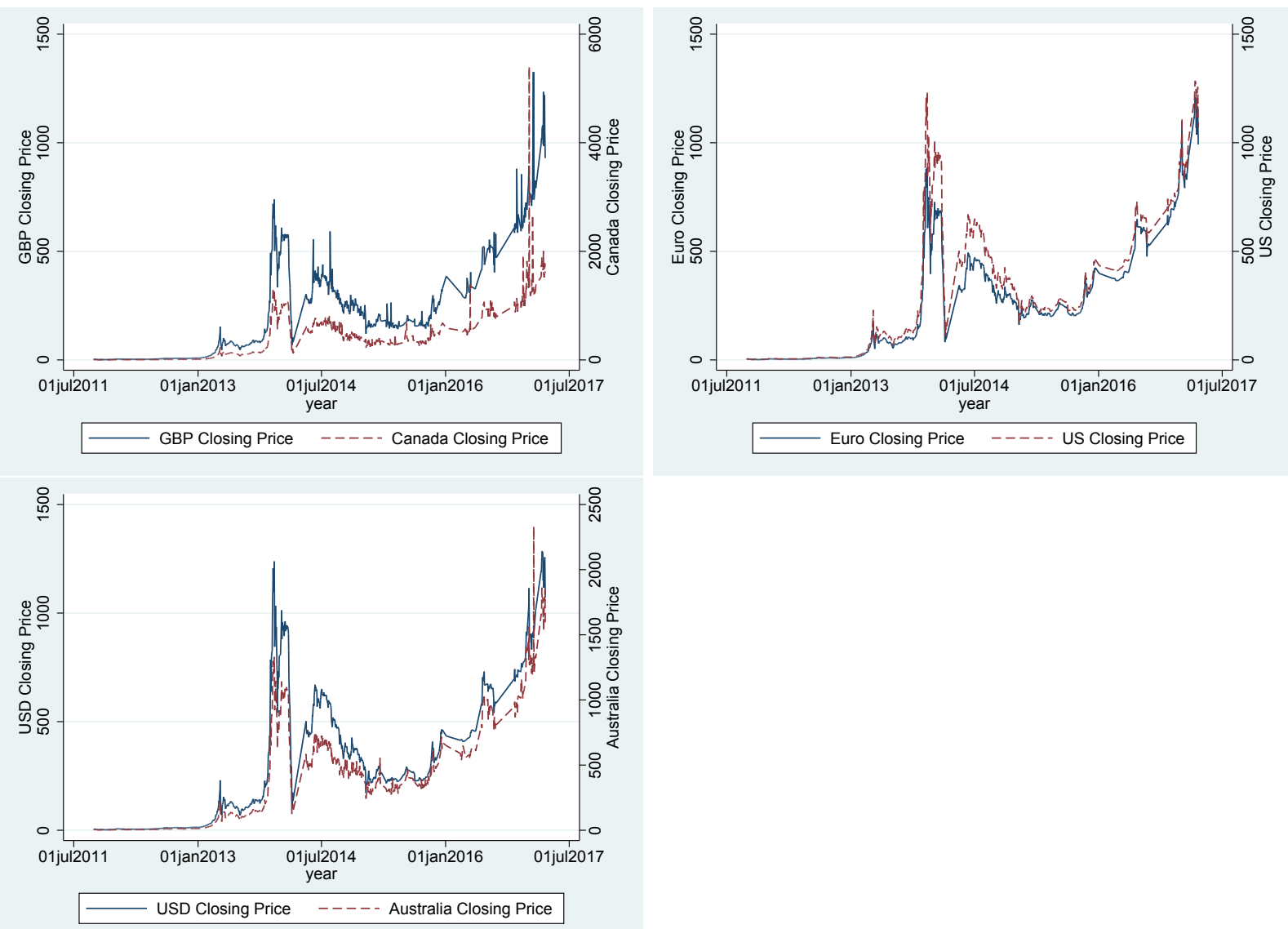
Figure 2: Autocorrelation functions

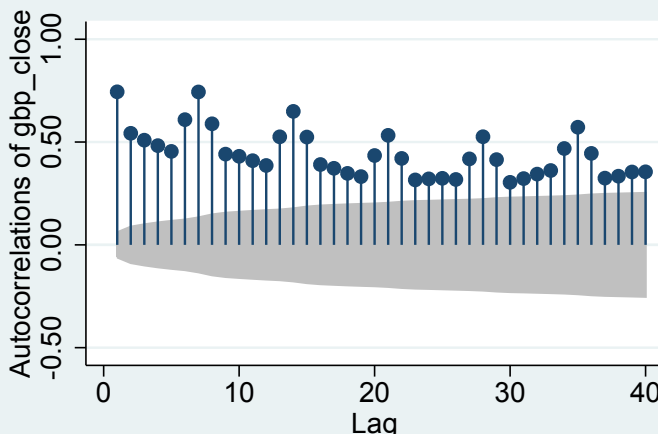

Bartlett's formula for MA(q) $95 \%$ confidence bands

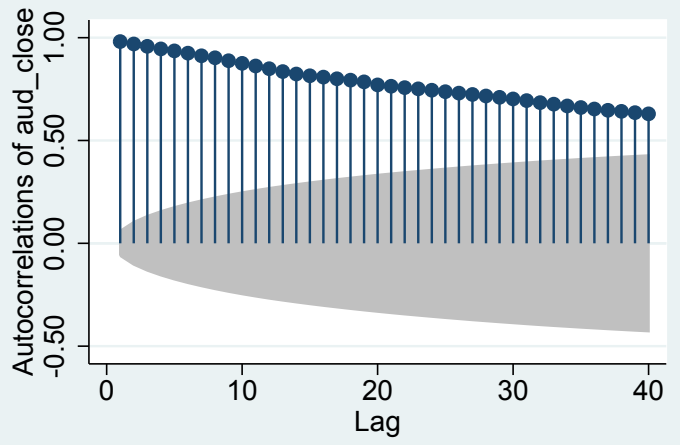

Bartlett's formula for MA(q) $95 \%$ confidence bands
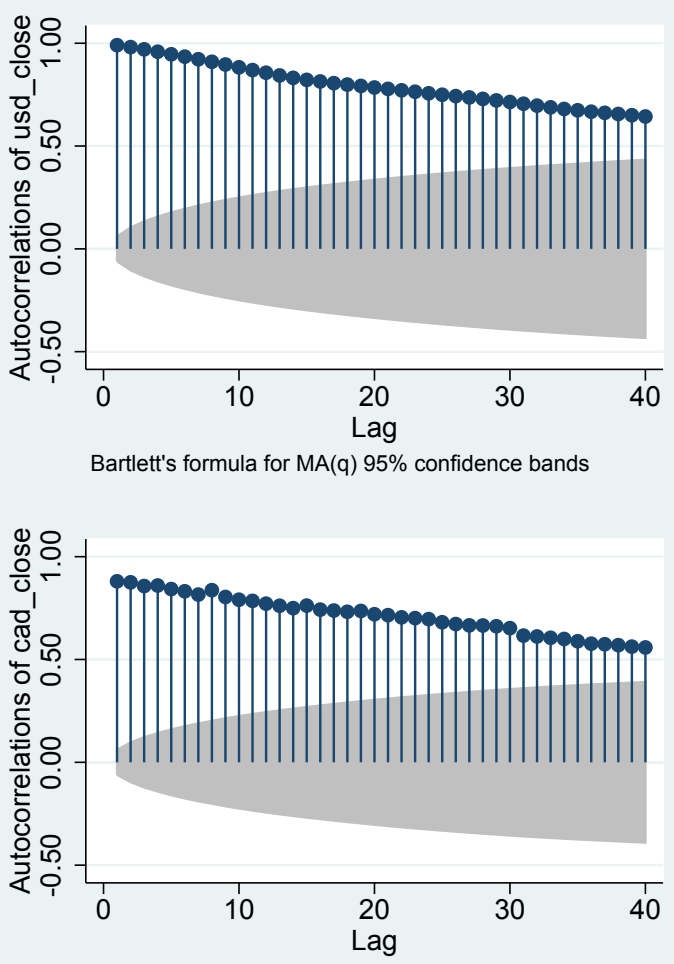

Bartlett's formula for MA(q) $95 \%$ confidence bands

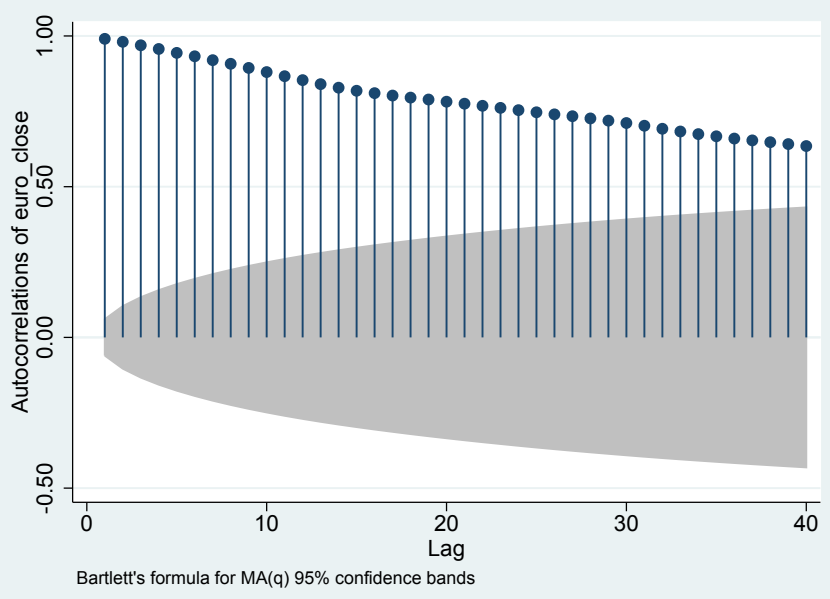


Figure 3: Estimated spectral density functions
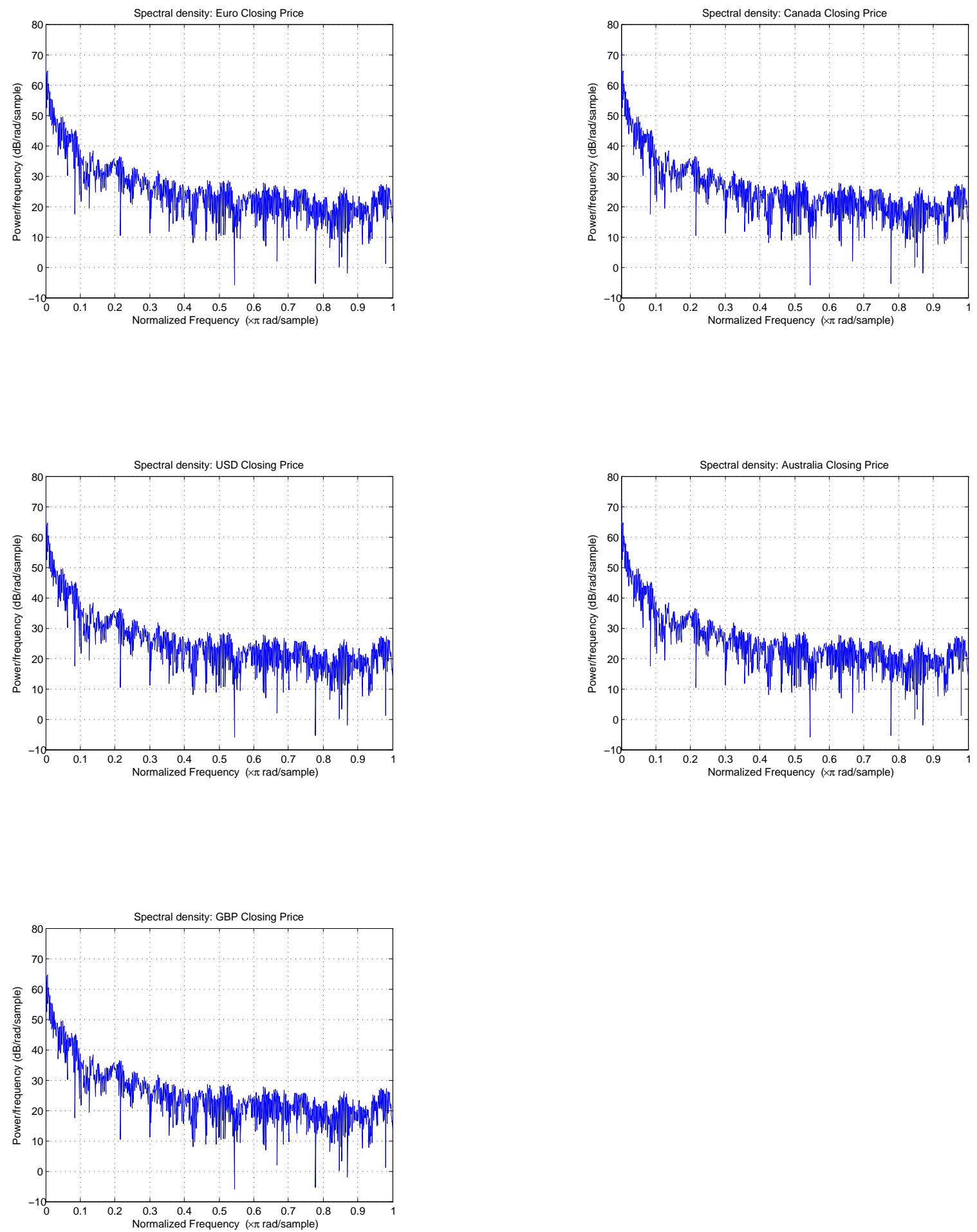